\title{
Erratum: Anatomy of Dzyaloshinskii-Moriya Interaction at Co/Pt Interfaces [Phys. Rev. Lett. 115, 267210 (2015)]
}

Hongxin Yang, André Thiaville, Stanislas Rohart, Albert Fert, and Mairbek Chshiev (Received 27 April 2017; published 24 May 2017)

DOI: 10.1103/PhysRevLett.118.219901

The DMI values reported in the manuscript should be multiplied by $\sqrt{3}$. This does not affect the results and all physical conclusions in the paper remain unchanged. 